\title{
NORMAL CORONARY DIAMETERS IN CORONARY ANGIOGRAM AT PATAN HOSPITAL, NEPAL
}

\author{
Ashok Adhikari, Kunal Bikram Shaha
}

Department of Internal Medicine (Cardiology), Patan Hospital, Patan Academy of Health Sciences, Lalitpur, Nepal

\begin{abstract}
This study aims to assess the normal coronary diameters of patient who underwent coronary angiogram in Patan Hospital. Angiographic and demographic data of a total of 307 patients (155 males, 152 females; mean age 62.09 \pm 11.06 years) who underwent elective coronary angiography in Patan Hospital due to suspicion of coronary artery disease between 2017 and 2020 and in whom coronary angiography documented normal coronary arteries without any intra-luminal irregularity were analyzed retrospectively. Proximal diameters of the main epicardial coronary arteries were measured quantitatively using automated software analysis (Allura, Philips). The mean diameter of unadjusted/adjusted left main coronary artery, proximal left anterior descending artery, proximal left circumflex artery, proximal right coronary artery were $4.87 \pm 0.85 \mathrm{~mm} / 2.8 \pm 0.54,3.8 \pm 0.7 / 2.19 \pm 0.439,3.4 \pm 0.7 / 1.98 \pm 0.44,3.6 \pm 0.85 / 2.07 \pm 0.53$ respectively. Our study findings contradict the traditional belief that females have narrower coronary arteries than males. Our study showed the females have statistically significant larger unadjusted Right Coronary Artery diameter and adjusted Left Main Coronary Artery diameter. We believe that our findings may contribute to the global data pool of normal coronary diameters and can be utilized in future studies as a database.
\end{abstract}

\section{KEYWORDS}

Heart, Coronary, Angiogram, Nepal

\section{CORRESPONDING AUTHOR}

Dr. Ashok Adhikari,

Department of Internal Medicine (Cardiology),

Patan Hospital, Patan Academy of Health Sciences,

Lalitpur, Nepal

Email: ashok.adhikari@gmail.com

Orcid No: https://orcid.org/0000-0001-5909-4409

DOI: https://doi.org/10.3126/nmcj.v23i3.40384 


\section{INTRODUCTION}

Cardiovascular diseases account for around one out of every three deaths worldwide. ${ }^{1}$ In Nepal, Coronary Artery Disease (CAD) is one of the common cardiovascular diseases seen by physicians in their hospital and private practice. Exact national data on incidence and prevalence of CAD in Nepal is not available. Smoking, dyslipidaemia, hypertension, diabetes, and physical inactivity are conventional risk factors. ${ }^{2}$ Therefore, acquiring a true notion of coronary anatomy is of paramount importance, including normal diameters of coronary arteries.

In healthy individuals, coronary dimensions are subjected to changes on the basis of various conditions such as sex differences, aging, racial/ ethnicity factors, body surface area (BSA), body weight, vasomotor tone, and left ventricular mass. ${ }^{3}$ Furthermore, formation of a reliable database for normal coronary diameters unadjusted and adjusted for age, sex, BSA, and racial factors may prove useful in the attempts to improve interventional cardiac procedures and coronary artery bypass grafting. The number of studies evaluating quantitative angiographic normal dimensions of coronary arteries and comparing these dimensions among sex and different racial/ethnic groups is scarce and relatively old.

In this study, we aimed to investigate the normal coronary artery diameters of the patient who underwent coronary angiogram in Patan Hospital for angina like symptoms more comprehensively in a larger population to reach a more reliable conclusion.

\section{MATERIALS AND METHODS}

We retrospectively analyzed the angiographic and demographic data of a total of 307 patients (155 males, 152 females; mean age 62.09 111.06 years) who underwent elective coronary angiography in Patan Hospital due to suspicion of coronary artery disease between 2017 and 2020 and in whom coronary angiography documented normal coronary arteries without any intra-luminal irregularities. Exclusion criteria were based on patient history, echocardiographic and coronary angiogram findings done during initial investigations as follow: moderate-to-severe stenotic or regurgitant valvular heart disease, history of acute coronary syndrome, evidence of localized or widespread coronary atherosclerosis, dilated or hypertrophic cardiomyopathy, severe renal failure, evidence of coronary vasospasm during the procedure, administration of nitroglycerine before image acquisition. This study was approved by Institutional Review Committee (IRC) of Patan Hospital.

Calculation of proximal coronary artery diameter: Coronary angiographies were performed via Allura, Philips through the transradial/transfemoral route using 5F/6F Judkins right and left or Tiger coronary diagnostic catheters. Standard projections recorded at 15 frames/sec were obtained from each patient. Proximal diameters of the main epicardial coronary arteries were measured quantitatively using automated software analysis (Axiom, Philips) Calibration for the coronary diameter was performed with reference to the catheter diameter. This quantitative angiography with the help of edge detection method is compatible with the previous studies. ${ }^{4,5}$ Location of the pertinent proximal coronary diameters for the measurement were as follows: mid-part of the left main coronary artery (LMCA), midpart of the proximal left circumflex artery (pLCx) between its origin and the first obtuse marginalis branch, mid-part of the proximal left anterior descending artery (pLAD) between its origin and the first diagonal branch, and $1.5 \mathrm{~mm}$ of the proximal right coronary artery (pRCA) after it was given off from the aorta. Right anterior oblique projections were used for the diameter calculation of LMCA, pLCx, pLAD, while left anterior oblique projection was used for pRCA. ${ }^{5}$ Furthermore, we also adjusted all the diameter and LMCA lengths for BSA of each relevant patient. In this regard, the Mosteller's formula was implemented in the calculation of BSA: BSA $(\mathrm{m} 2)=$ square root of (Height $(\mathrm{cm}) \mathrm{X}$ Weight $(\mathrm{kg}) / 3600)$. The average values of both crude and adjusted proximal coronary diameters were compared separately.

STATISTICAL ANALYSIS: Primary data were used to carry out the research study. Descriptive cross-sectional study was done by taking 307 sample using Cochran's formula by taking margin of error $5 \%$. The data were recorded by using clinical observations. The recorded data were entered in to Microsoft Excel Version 2010 and then data editing, cleaning and validation was properly done. The data after editing was imported in to Statistical Packages for Social Sciences version 20. The assumption of normality was tested before applying statistical test. Since, the assumption of normality was not hold true for parametric t test, the nonparametric test Wilcoxon Rank sum test was performed in order to compare mean values of various coronary arteries parameters. 


\section{RESULTS}

Demographic and clinical characteristics of the patient are presented in Table 1 . The average age of the patient was $62.09 \pm 11.06$ years old and the total number of male and female were 155 and 152 respectively. Average weight of the patient was $71.14 \pm 8.41 \mathrm{~kg}$ and average height was $161.2 \pm 4.24 \mathrm{~cm}$. The mean BSA was $1.75 \pm 0.12 \mathrm{~m}^{2}$. Cardiovascular risk factor, in order of frequency were hyperlipidemia (85\%), hypertension (75.89\%), and diabetes mellitus
(45\%). For smoking category, never smoker (15.3\%), environmental tobacco smoker (4.2\%), someday smoker (18.6\%), current smoker (8.80\%), former smoker (48.9\%), and quit attempt (4.2\%). Of 307 patients, 279 (90.9\%) had right coronary dominance, $19(6.2 \%)$ had left coronary dominance and $9(2.9 \%)$ had codominance.

Table 2 shows the total and gender specific unadjusted and adjusted proximal coronary artery diameters. The unadjusted diameter

\begin{tabular}{|c|c|c|c|}
\hline Description & Frequency & Percentage & Mean \pm SD \\
\hline \multicolumn{4}{|l|}{ Age Group ( in years) } \\
\hline $29-39$ & 2 & 0.65 & \\
\hline $40-49$ & 38 & 12.38 & \\
\hline $50-59$ & 83 & 27.04 & $62.09 \pm 11.06$ \\
\hline $60-69$ & 93 & 30.29 & \\
\hline $70-79$ & 78 & 25.41 & \\
\hline $80-89$ & 12 & 3.91 & \\
\hline \multicolumn{4}{|l|}{ Gender } \\
\hline Male & 155 & 50.49 & \\
\hline Female & 152 & 49.51 & \\
\hline Height Group(cm) & & & $(161.2 \pm 4.24) \mathrm{cm}$ \\
\hline$<161 \mathrm{~cm}$ & 141 & 45.93 & \\
\hline$>=161 \mathrm{cms}$ & 166 & 54.07 & \\
\hline Weight Group(kg) & & & $(71.14 \pm 8.41) \mathrm{kg}$ \\
\hline$<72 \mathrm{~kg}$ & 145 & 47.23 & \\
\hline$>=72$ & 162 & 52.77 & \\
\hline $\operatorname{BSA}\left(\mathbf{m}^{2}\right)$ & & & $1.75 \pm 0.12$ \\
\hline \multicolumn{4}{|l|}{ Comorbidities } \\
\hline Hypertension & 233 & 75.89 & \\
\hline Diabetes Mellitus & 138 & 45.00 & \\
\hline Hyperlipidemia & 261 & 85.00 & \\
\hline \multicolumn{4}{|l|}{ Smoking } \\
\hline Never smoker & 47 & 15.30 & \\
\hline Environmental Tobaccosmoker(ETS) & 13 & 4.20 & \\
\hline Someday smoker & 57 & 18.60 & \\
\hline Current smoker & 27 & 8.80 & \\
\hline Former smoker & 150 & 48.90 & \\
\hline Quit attempt & 13 & 4.20 & \\
\hline \multicolumn{4}{|l|}{ Coronary Artery Dominance } \\
\hline Left Dominance & 19 & 6.20 & \\
\hline RT dominance & 279 & 90.90 & \\
\hline Codominance & 9 & 2.90 & \\
\hline
\end{tabular}


Table 2: Comparison of crude and BSA-adjusted proximal coronary artery diameters between genders

\section{Description}

LMCA diameter(mm)
Adjusted-LMCA diameter $\left(\mathrm{mm} / \mathrm{m}^{2}\right)$
LAD diameter
Adjusted-LAD diameter $\left(\mathrm{mm} / \mathrm{m}^{2}\right)$
LCx Diameter
Adjusted-LCx diameter $\left(\mathrm{mm} / \mathrm{m}^{2}\right)$
RCA
Adjusted-RCA diameter $\left(\mathrm{mm} / \mathrm{m}^{2}\right)$

\begin{tabular}{cccc}
$\begin{array}{c}\text { Total } \\
\text { population } \\
(\mathbf{n}=\mathbf{3 0 7 )}\end{array}$ & $\begin{array}{c}\text { Male } \\
\text { Gender } \\
\mathbf{n}=\mathbf{1 5 5} \\
\mathbf{M e a n} \pm \mathbf{S D}\end{array}$ & $\begin{array}{c}\text { Female } \\
\text { Gender } \\
\mathbf{n}=\mathbf{1 5 2} \\
\mathbf{M e a n} \pm \mathbf{S D}\end{array}$ & $\begin{array}{c}\mathbf{P} \\
\text { value }\end{array}$ \\
$4.87 \pm 0.85$ & $4.86 \pm 0.81$ & $4.89 \pm 0.88$ & 0.189 \\
$2.8 \pm 0.54$ & $2.76 \pm 0.54$ & $2.84 \pm 0.55$ & 0.032 \\
$3.8 \pm 0.7$ & $3.78 \pm 0.65$ & $3.84 \pm 0.76$ & 0.648 \\
$2.19 \pm 0.43$ & $2.14 \pm 0.42$ & $2.23 \pm 0.46$ & 0.074 \\
$3.4 \pm 0.7$ & $3.43 \pm 0.65$ & $3.45 \pm 0.76$ & 0.534 \\
$1.98 \pm 0.44$ & $1.95 \pm 0.41$ & $2.009 \pm 0.46$ & 0.577 \\
$3.6 \pm 0.85$ & $3.61 \pm 0.797$ & $3.61 \pm 0.91$ & 0.001 \\
$2.07 \pm 0.53$ & $2.05 \pm 0.48$ & $2.08 \pm 0.57$ & 0.797 \\
\hline
\end{tabular}

BSA: Body surface area; SD: Standard deviation; LMCA: Left main coronary artery; LAD: Left anterior descending artery; LCx: Left circumflex artery; RCA: Right coronary artery

of LMCA, LAD, and LCX were larger in female compared to male. However, these data were statistically not significant $(\mathrm{P}>0.05)$. Furthermore, when these unadjusted diameter was adjusted for BSA, the diameter of adjusted LAD, LCX and RCA were again larger in female and these data were also statistically not significant $(\mathrm{P}>0.05)$.

Accordingly, unadjusted RCA diameter and adjusted LMCA diameter were also larger in female and however these data were found to be statistically significant $(<0.05)$.

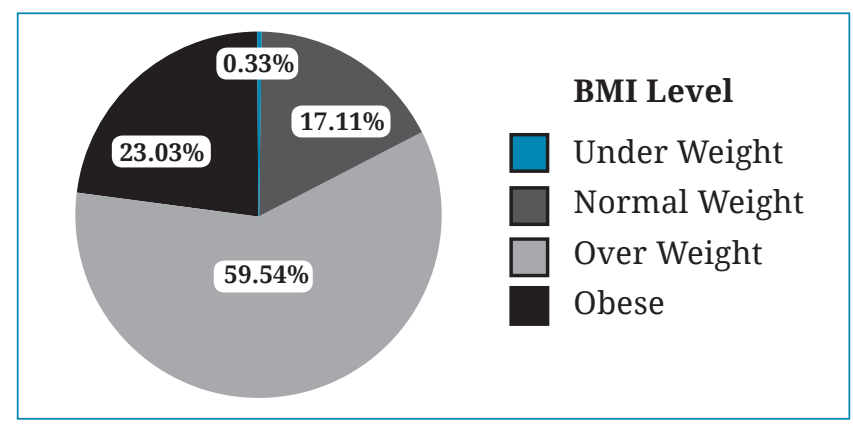

Fig. 1: Pie Chart showing percentage of population of different BMI level

Out of 307 patients $0.33 \%$ was under weight, $17.11 \%$ were of normal weight, $59.54 \%$ were overweight and $23.03 \%$ were obese (Fig. 1).

\section{DISCUSSION}

The dimensions of the coronary arteries are highly variable in the normal population. The determinants of coronary artery size are not well understood. Genetic factors undoubtedly play an important role. Age, sex, body weight, BSA, weight of the heart, and ethnic/racial factors have all been correlated with the coronary artery anatomy in various studies. ${ }^{6}$ Most previous studies evaluated human coronary artery lumen diameter in cadaveric hearts, and there have been very few reports regarding the estimation of the coronary artery dimensions by a quantitative angiographic method during clinical practice. There have been very few estimates of normal coronary artery size in real life based on visual estimates or electronic caliper measurements from cineangiographic films. ${ }^{7}$ In this retrospective study we attempted to establish a database for normal dimensions of the coronary artery segments by using quantitative coronary angiography

In this study the principles of quantitative coronary angiography for assessment of angiographically normal coronary artery segments in 307 patients who had undergone cardiac catheterization and angiography for evaluation of symptoms suggestive of coronary artery disease. The majority of the patient were among the age group of 50-80 years old. Male and female were almost equal in numbers with average weight of $71.14 \pm 8.41 \mathrm{~kg}$ and average height of $161.2 \pm 4.24 \mathrm{~cm}$. The cardiovascular risk factor, in order of frequency were hyperlipidemia (85\%), hypertension (75.89\%), and diabetes mellitus (45\%). For smoking category: never smoker (15.3\%), environmental tobacco smoker (4.2\%), someday smoker (18.6\%), current smoker (8.80\%), former smoker (48.9\%), quit attempt (4.2\%). Of 307 patients, $279(90.9 \%)$ had right coronary dominance, 19(6.2\%) had left coronary dominance and 9 (2.9\%) had codominance.

In this study, the mean diameter of unadjusted/ adjusted proximal LMCA, LAD, LCX and RCA were $4.87 \pm 0.85 \mathrm{~mm} / 2.8 \pm, \quad 3.8 \pm 0.7 / 2.19 \pm 0.439$, 
$3.4 \pm 0.7 / 1.98 \pm 0.44$ and $3.6 \pm 0.85 / 2.07 \pm 0.53$, respectively. It was found that the unadjusted diameter of LMCA, LAD, LCX were larger in female compared to male. However, these data were statistically not significant $(P>0.05)$. Moreover, when these unadjusted diameter were adjusted for BSA, the diameter of adjusted LAD, LCX and RCA were again larger in female and these data were also statistically not significant $(\mathrm{P}>0.05)$. Accordingly, unadjusted RCA diameter and adjusted LMCA diameter were also larger in female and these data were found to be statistically significant $(\mathrm{P}<0.05)$. These findings might be due to our study population were diseased in one way or another as they were visiting hospital for health related issues although their coronary angiogram results were insignificant. Large study in community with healthy individual might show different results.

This finding contradicts the traditional belief of females having smaller coronary artery compared to males. Our finding differs from the finding of Elagovan et al. ${ }^{8}$ who have found that females have smaller dimension of coronary artery after correction for BSA.Our results were consistent with those of previous studies, which showed that the diameters of coronary arteries are highly variable, considering that genetic and environmental factors including age, sex, ethnicity, and race have all been correlated with the coronary artery diameter. ${ }^{9}$ Previous reports have also indicated a gender-related difference in coronary artery diameters. ${ }^{10}$

To the best of our knowledge, our study seems to be the only study with a larger Nepalese population where both of adjusted and unadjusted proximal coronary diameters were compared. The clinical and therapeutic implications of a narrow or wide coronary artery is obvious. A moderate denovo plaque would result in significant occlusion in smaller vessels and the burden of atheroma required for the development of significant coronary lesions is lower in smaller arteries. Evidence suggests that acute or subacute stent occlusion or thrombosis is more common in vessels less than $2.5 \mathrm{~mm}$ in diameter. Smaller coronary arteries may result in technical difficulties during vascular procedures such as coronary artery bypass or percutaneous coronary interventions.

Our study findings contradict the traditional belief that females have narrower coronary arteries than men. Our study showed the females have statistically significant larger unadjusted RCA diameter and adjusted LMCA diameter. We believe that our findings may contribute to the global data pool of normal coronary diameters and can be utilized in future studies as a database. This has implications for predisposition to atherosclerosis, and more challenging performance of procedures such as coronary artery bypass grafting, stent implantation, or atherectomy. Future cohort studies with adequate sample sizes are needed to confirm the results of our study.

\section{ACKNOWLEDGEMENT}

We would like to thank the catheterization laboratory, Medicine Department and IRC, Patan Academy for health science for the permission provided for this study.

Conflict of interest: None

Source of research fund: None

\section{REFERENCES}

1. GBD 2015 Eastern Mediterranean Region Cardiovascular Disease Collaborators. Burden of cardiovascular diseases in the Eastern Mediterranean Region, 1990-2015: findings from the Global Burden of Disease 2015 study. Int'l J Public Health 2018; 63: 137-49.

2. Maskey A, Sayami A, Pandey MR. Coronary Artery Disease: An Emerging Epidemic In Nepal. J Nepal Med Assoc 2003; 42: 122-4.

3. Dodge JT Jr, Brown BG, Bolson EL, Dodge HT. Lumen diameter of normal human coronary arteries. Influence of age, sex, anatomic variation, and left ventricular hypertrophy or dilation. Circulation 1992; 86: 232-46.

4. Raut BK, Patil VN, Cherian G. Coronary artery dimensions in normal Indians. Indian Heart $J$ 2017; 69: 512-4.

5. Lip GY, Rathore VS, Katira R, Watson RD, Singh SP. Do Indo-Asians have smaller coronary arteries? Postgrad Med J 1999; 75: 463-6.

6. Dodge J, Greg Brown B, Bolson Edward L, Harold T. Dodge lumen diameter of normal human coronary arteries: influence of age, sex, anatomic variation, and left ventricular hypertrophy or dilation. Circulation 1992; 86: 232-46.

7. Dhawan J. Are Asian coronary arteries smaller than Caucasian: a study on angiographic coronary artery size estimation during life. Int'l J Cardiol 1995; 49: 267-9.

8. Elangovan C, Jaganathan V, Alageshan R. Clinical and anthropometric correlation of normal Coronary artery dimensions. Indian Heart J 2005; 57: 381-425.

9. Wilsens SL, Plair CM, Henderson D. Size of the major epicardial coronary arteries at necropsy. Relation to age, heart weight, and myocardial infarction. J Amer Med Assoc 1966; 198: 1325-9.

10. Mahadevappa M, Hegde M, Math R. Normal proximal coronary artery diameters in adults from India as assessed by Computed Tomography angiography. J Clin Diagn Res 2016; 10: 10-3. 